\title{
A Study To Assess The Effectiveness Of Deep breathing Exercise With Incentive Spirometer On The Respiratory Status Of Patients Who Have Undergone Cardio Thoracic And Vascular Surgery In Selected Hospital Puducherry.
}

${ }^{*}$ Ms. Aileen George ${ }^{* *}$ Dr.Renuka.K\& ${ }^{* * *}$ Mrs. Kripa Angeline.A

\section{Abstract:}

Objectives: The main aim of the study was to assess the effectiveness of deep breathing exercise with incentive Spirometer on the respiratory status of patients who have undergone cardio thoracic and vascular surgery in selected hospitals of Puducherry. Methods: Research design used for the study was quasi experimental one group pre-test post-test design. The study was conducted on 30 patients who have undergone cardio thoracic and vascular surgery at Cardio Thoracic and Vascular wards of Mahatma Gandhi medical College and Research Institute (MGMC\&RI), Puducherry and A G Padmavathy hospital, Puducherry. Stratified random sampling technique was used. Data collection tool consisted of demographic variables and assessment of respiratory status using Respiratory Rate, Modified Borg Dyspnea Scale, Peak Flow Meter and Pulseoxymeter. Data were collected by pre assessment questionnaire and observation method. The collected data was tabulated, analyzed and interpreted by using descriptive and inferential statistics. Result: The result showed that deep breathing exercise with incentive spirometer was highly effective among patients who have undergone cardio thoracic and vascular surgery. The obtained P-value 0.000 was highly significant at $p<0.001 \%$ level. Conclusion: The respiratory complications are the major post-operative complications which reduce the projected outcome of most of the cardio thoracic and vascular surgeries. This study reveals that deep breathing exercise with incentive spirometer can improve the respiratory status of the patients who have undergone cardio thoracic vascular surgery to a great extent.

Key words: Deep Breathing Exercise, Incentive Spirometer, , and Respiratory Status.

\section{INTRODUCTION}

Cardio thoracic and vascular surgeries are getting important for the management of many cardiac and pulmonary disorders.United Nations Demographic year book (2008) states that more than 4 million surgeries are done worldwide.
Among that about $30 \%$ of them are cardio thoracic and vascular surgeries ${ }^{1}$.The Annual Health Indices (2010) shows that about 0.5 million Cardio Thoracic and Vascular Surgeries are done yearly in India. Out of them $50 \%$ to $70 \%$ is Coronary Artery Bypass Grafting2 ${ }^{2}$ Even though most of these surgeries are successful, some of them are not free of post-operative 
complications. One of the most occurred post-operativecomplication is pulmonary complication which can adversely affect the outcome .Among the 0.5 million cardio vascular and thoracic surgery done in India one of the most worrisome complication is decline in pulmonary function. Such complications can be avoided with the administration of simple measures like deep breathing exercise along with incentive spirometry which can reduce the occurrence of pulmonary complications as well as improve the respiratory status of the patient3. Urell C and et al (2007) conducted a study at Denmark which tells that in addition to early mobilization, a variety of breathing exercises are used to prevent postoperative pulmonary complications after cardiac surgery 4

\section{NEED FOR THE STUDY}

With the epidemiologic transition the Cardio Vascular Disease burden continues to rise in developing countries including India. The projected rise in disease burden due to Cardio Vascular Disease is expected to make it the prime contributor of total mortality and morbidity. About 2.3 million people in India are suffering from different cardio vascular diseases and $44.3 \%$ of them need angiogram for evaluation and $26.3 \%$ of them need CABG as intervention 5

\section{WHO Annual World Health}

Statistics of the year 2010 shows that about fifty million people are suffering from cardiovascular problems globally and about $30-60 \%$ of them are undergoing for surgical interventions. With the increasing incidence of cardiac and respiratory diseases, the surgical interventions to treat these problems are also hovering.

Andrew et al (2008) stated that chest therapy after surgery is directed towards maximal inspiration in an attempt to prevent overt atelectasis and allow for the early re-expansion of collapsed alveoli. Adoption of incentive spirometer as a global method of prophylaxis, however, raises concerns that high risk patients may be receiving inadequate treatment and that important resources are being wasted on low risk patients. The objective of this trial was to evaluate the prevention of respiratory complications by comparing a global policy of incentive spirometer with a regimen consisting of deep breathing exercises for low risk patients and incentive spirometer plus physiotherapy for high risk patients. So they suggested deep breathing exercise along with incentive spirometer as the cheapest method for improving the respiratory status

\section{OBJECTIVES}

1. To assess the respiratory status of patients who have undergone cardio thoracic and vascular surgery during pretest.

2. To assess the effectiveness of deep breathing exercise with incentive spirometer on the respiratory status of patients who have undergone cardio thoracic and vascular surgery during post test.

3. To find out the association between effectiveness of deep breathing exercise and incentive spirometer on respiratory status of patients who have undergone cardio thoracic and vascular surgery and selected demographic variables.

\section{NULL HYPOTHESES}

$\mathrm{H}_{01}$-There is no significant difference between the pretest and post test respiratory status of the patients who have under gone cardio thoracic and vascular surgery before and after the administration of deep breathing exercise with incentive spirometer. 
$\mathbf{H}_{02}$-There is no significant association between the effectiveness of deep breathing exercise with incentive spirometer on respiratory status of patients who have undergone cardio thoracic and vascular surgery and demographic variables.

\section{RESEARCH METHODOLOGY}

Quantitative Research approach was used \&the research design for this study was Quasi experimental one-group pre-test and post-test design.The study was conducted in Cardio Thoracic and Vascular surgery wards of Mahatma Gandhi Medical College and Research Institute (MGMC\&RI), Puducherry and A G Padmavathy hospital, Puducherry. The target population of the present study was patients who have undergone for cardio thoracic and vascular surgery. The samples of the study were patients who have undergone for Cardio Thoracic and Vascular Surgery at Mahatma Gandhi Medical College and Research Institute, Puducherry and A G Padmavathy hospital, Puducherry. 60 Sample were selected by stratified random sampling technique. The tool for data collection included Demographic variables\& Assessment of respiratory status using Respiratory Rate, Modified Borg Dyspnea Scale, Peak Flow Meter and Pulseoxymeter.

PART B:Assessment of respiratory status using Respiratory Rate, Modified Borg Dyspnea Scale, Peak Flow Meter and Pulseoxymeter.

\section{Respiratory Rate}

a) <12 breaths/minute- bradypnea

b) $12-22$ breaths/minute- normal

c) $>22$ breaths/minute- tachypnea

\section{Modified Borg Dyspnea Scale}

a) Grade I- slight breathlessness

b) Grade II- moderate breathlessness

c) Grade III- severe breathlessness

d) Grade IV- maximum breathlessness
III. Peak Flow Meter

a) $81-100 \%$ - free of symptoms

b) $51-80 \%$ - caution

c) $<50 \%$ - danger

IV. Pulseoxymeter

a) $91-100 \%$ oxygen saturation- normal

b) $81-90 \%$ oxygen saturationdesaturated

c) $<80 \%$ oxygen saturation- hypoxic

\section{SCORE INTERPRETATION}

Part $B$ is the assessment of respiratory status with the help of Respiratory Rate, Modified Borg Dyspnea Scale, Peak Flow Meter and Pulseoxymeter.

The criteria for respiratory assessment are adequate and inadequate.

\section{DATA COLLECTION PROCEDURE}

The pretest was done on the

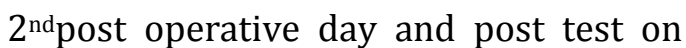
the $7^{\text {thpost }}$ operative day with the respiratory status assessment parameters like Respiratory Rate, Dyspnea using Modified Borg Dyspnea Scale, Flow rate using Peak Flow Meter and Pulseoxymeter. After finishing the intervention for 5 days i.e. on the $7^{\text {th }}$ post operative day, the investigator had assessed the respiratory status of the subjects with the respiratory status assessment parameters

\section{RESULTS:}

The mean difference was 0.60 between pretest mean score (1.63) and post test mean score (1.03). The table shows that there was decrease in standard deviation values from pretest to post test. The obtained paired ' $\mathrm{t}$ ' value was 6.595 and P-value was $0.000^{* * *}$. It was highly significant at $\mathrm{p}<0.001 \%$ level.It was inferred that deep breathing exercise with incentive spirometer is effective in improving the respiratory status of the patients who have under gone cardio thoracic and vascular surgery. 
$T A B L E-1$

Effectiveness of incentive Spirometer on the respiratory status of patients who have under gone cardio thoracic and vascular surgery.

\begin{tabular}{|c|c|c|c|c|c|c|}
\hline Group & Mean & Mean Difference & $N$ & $S D$ & $\begin{array}{l}\text { 't'value } \\
\text { (df) }\end{array}$ & Pvalue \\
\hline Pretest & 1.63 & \multirow{2}{*}{0.60} & 30 & 0.490 & \multirow{2}{*}{$\begin{array}{c}6.595 \\
(29)\end{array}$} & \multirow{2}{*}{$0.000^{\ldots}$} \\
\hline Post test & 1.03 & & 30 & 0.183 & & \\
\hline
\end{tabular}

\section{DISCUSSION}

The study finding showed that mean difference was 0.60 between pretest mean score (1.63) and post test mean score (1.03). The table shows that there was decrease in standard deviation values from pretest to post test.

The obtained paired ' $\mathrm{t}$ ' value was 6.595 and P-value was $0.000^{* * *}$. It was highly significant at $\mathrm{p}<0.001 \%$ level. It was inferred that deep breathing exercise with incentive spirometer is effective in improving the respiratory status of the patients who have under gone cardio thoracic and vascular surgery.

With regard to the effectiveness 0 deep breathing exercise with incentive spirometer on respiratory status of patients who have undergone cardio thoracic anc vascular surgery, the result shows that deer breathing exercise with incentive spirometer was highly effective among patients whe have undergone cardio thoracic and vascular surgery. The obtained P-value 0.000 was highly significant at $\mathrm{p}<0.001 \%$ level. Hence $\mathrm{H}_{01}$ was rejected.

Regarding the association between the effectiveness of deep breathing exercise with incentive spirometer on the respiratory status of patients who have undergone cardio thoracic and vascular surgery and demographic variables, the result revealed that there was no significant association. Hence H02 was rejected

\section{RECOMMENDATIONS}

- A longitudinal study can be conducted to evaluate the changes happening in the respiratory status with deep breathing exercise and incentive spirometer.

* A comparative study can be conducted to assess the effectiveness of purse lip breathing and diaphragmatic breathing on respiratory status using a large number of samples

\section{CONCLUSION:}

The respiratory complications are the major post-operative complications which reduce the projected outcome of most of the cardio thoracic and vascular surgeries. This study reveals that deep breathing exercise along with incentive spirometer can improve the respiratory status of the patients who have undergone cardio thoracic vascular surgery to a great extent. Many of the respiratory complications can be avoided with proper respiratory management.. With little effort it can be implemented on all the postoperative patients for better respiratory status and for the prevention of respiratory complications 


\section{REFERENCES:}

1. Black M J, Hawks. Medical-Surgical Nursing: Clinical management for positive outcomes.7thed. New Delhi: Elseiver; 2004.

2. Boon M. Davidson. Principles and Practice of Medicine. 20th ed. Philadelphia: FA Davis Company; 2006.

3. Burns Nancy Understanding Nursing Research. 3rd ed. London: WB Saunders Company; 1999.

4. Burns $\mathrm{N}$ and Grove $\mathrm{S}$ K. The practice of Nursing Research, Conduct, Critique, and Utilization .8th ed. Missouri:Elseiver; 2005.
5. Gleora Barbara Kozier. Fundamentals of Nursing.7th ed. New Delhi: Pearson Education; 2004.

6. Kumar V, Abbas A K., Fausto N. Robbins and Cotran Pathologic basis of disease. 7thed. New Delhi: Elseiver; 2004

7. Andrew "evaluate the prevention of respiratory complications by comparing a global policy of incentive spirometer with a regimen consisting of deep breathing exercises for low risk patients and incentive spirometer plus physiotherapy for high risk patients

\section{SYMPTOMS OF HYPOXIA}

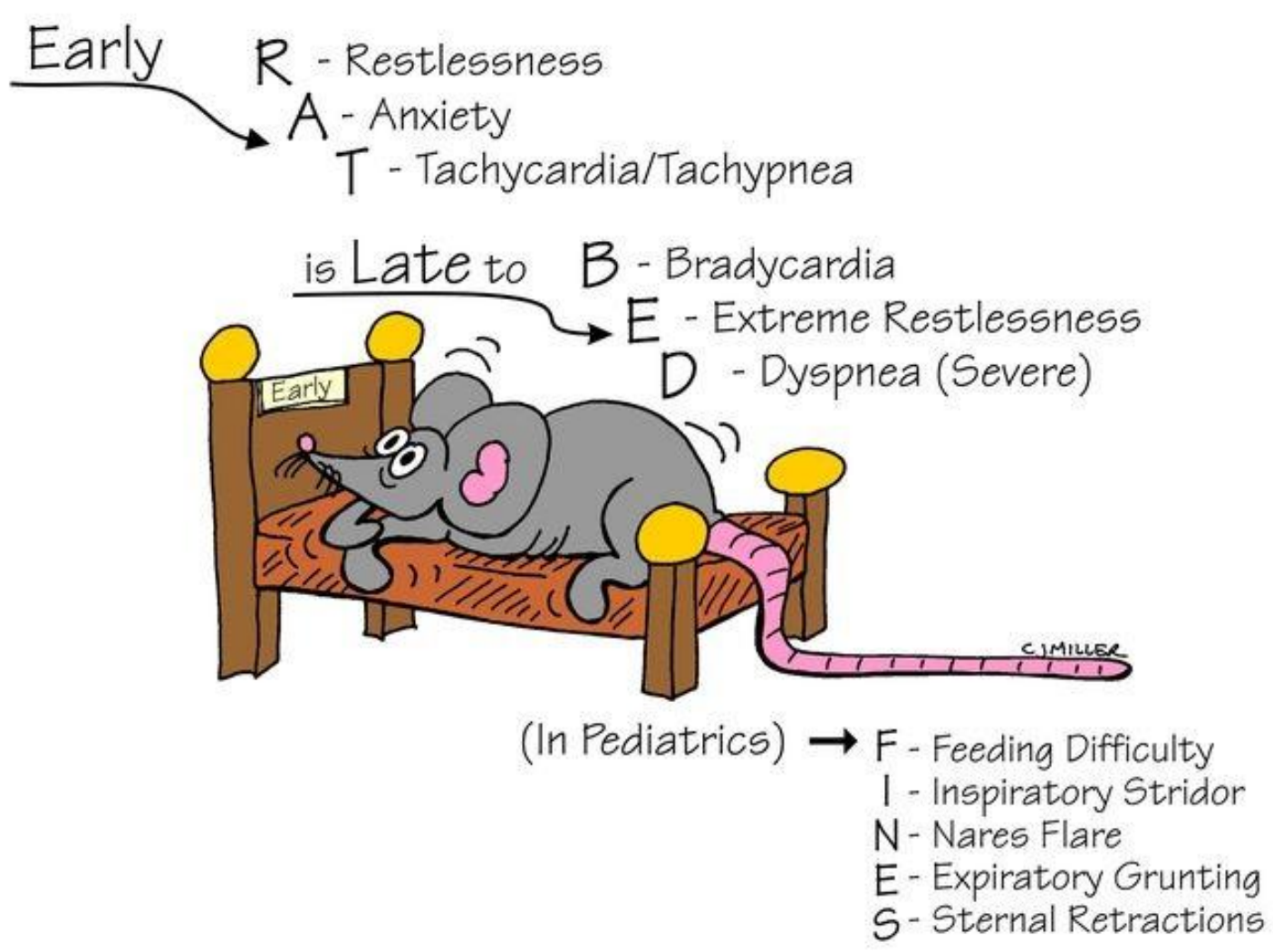

